\title{
Tratamiento quirúrgico de las úlceras por presión sacras con colgajo miocutáneo de gluteus maximus por deslizamiento en V-Y: Técnica quirúrgica
}

\author{
Wilfredo Calderón O. ${ }^{1}$, Kenneth Guler G. ${ }^{1}$, Juan Pablo Camacho M. ${ }^{1}$, Juan José Lombardi A. ${ }^{1}$, \\ Miguel Obaid G. ${ }^{1}$ y Rodrigo Subiabre F. ${ }^{1}$
}

'Departamento de Cirugía Sede Oriente, Universidad de Chile - Hospital del Salvador. Santiago, Chile.

Recibido 2020-11-17, aceptado 2021-08-03

Correspondencia a: Dr. Wilfredo Calderón Ortega wilfredocalder@gmail.com
Surgical treatment of sacral pressure ulcers with gluteus maximus myocutaneous flap due to V-Y sliding: surgical technique

Introduction: Pressure sores are the result of the compression of soft tissues in the prominent bones areas, mainly in patients without movement. If the depth of the ulcer compromises the sacral bone, the treatment will be the gluteus maximus myocutaneous flap in V-Y. Materials and Method: In the plastic surgery service of the hospital del Salvador 82 patients with sacral pressure sores grade 4 were operated on between 2011 and 2020 with gluteus maximus myocutaneous V-Y flap. The last 37 patients were analyzed. Results: With this treatment the flaps were doing well in all cases with good blood supply. Complications: dehiscence: 2 patients, cellulitis: 2 patients, hematoma: 2 patients and seroma: 1 patient. The overall complication was $16 \%$. Follow up between three and six months. Discussion: The most important part in pressure sores is their prevention. When the ulcer is in prominent parts of the body, the sore, could be in different grades of depth. The classification of them is in grades 1: erythema, 2: subcutaneous tissue. These two grades are solved with conservative treatment. When the ulcers are in grade 3 or 4 , and with little ulcer in the skin but with damage of the deep plane, the treatment will be with surgery. In our casuistic the most frequent pressure sore is in the sacrum treated with gluteus maximus sliding myocutaneous flap in V-Y. With this treatment the flaps were doing well in all cases with good blood supply. The complications of $16 \%$ were considered low. Conclusion: The sliding gluteus Maximus myocutaneous flap in V-Y for treatment for sacral pressures sores have been successful for our patients.

Keywords: reconstructive surgery; pressure ulcer; myocutaneous flap.

\section{Resumen}

Introducción: La úlcera por presión más frecuente es la sacra. Si compromete el hueso puede provocar osteomielitis por lo que requiere aseo quirúrgico y colgajo miocutáneo de gluteus maximus por deslizamiento en V-Y. Materiales y Método: En el servicio de cirugía plástica del hospital del Salvador entre 2011 y 2020 se han operado 82 pacientes con ulceras sacras grado 4 que requirieron cobertura con colgajo miocutáneo de gluteus maximus en V-Y. De ellas se analizaron los últimos 37 pacientes. Resultados: De los 37 pacientes, 12 fueron mujeres, 25 hombres, 17 parapléjicos, 12 tetrapléjicos y 8 deambulaban. 6 pacientes presentaron COVID-19. 24 fueron bilaterales y 13 unilaterales. Los colgajos cicatrizaron bien en 30 pacientes. Las complicaciones fueron de $16 \%$ consistente en 2 hematomas, 2 dehiscencias, 2 celulitis y 1 seroma, todas resueltas sin problemas. Seguimiento de entre 3 y 6 meses. Discusión: El tratamiento quirúrgico con colgajo miocutáneo de gluteus maximus en V-Y, aparte de aportar volumen para ocluir la úlcera, aporta irrigación excelente con oxígeno, nutrientes y antibióticos que aseguran una óptima cicatrización. Dependiendo del diámetro de la úlcera el colgajo puede ser uni o bilateral. Dado lo complejo del tratamiento, en general, el porcentaje de complicaciones de $16 \%$ se considera bajo. Conclusión: El tratamiento quirúrgico de las úlceras por presión sacras con colgajos miocutáneos deslizantes de gluteus maximus ha sido exitoso con buenos resultados quirúrgicos con buen flujo sanguíneo y buena evolución.

Palabras clave: cirugía reconstructiva; úlceras por presión; colgajos miocutáneos. 


\section{Introducción}

Las úlceras por presión son lesiones de los tejidos blandos, resultantes de la presión prolongada de la piel entre una prominencia ósea y el lugar de apoyo.

Cuando la presión externa a la piel sobrepasa la presión capilar, el flujo sanguíneo se interrumpe. La presión capilar venosa es de 12 milímetros de mercurio (mm de $\mathrm{Hg}$ ) y de hasta $32 \mathrm{~mm}$ de $\mathrm{Hg}$ en la fase arterial. Si se sobrepasa esos límites sobreviene la isquemia. Presiones sobre $60 \mathrm{~mm}$ de Hg por más de dos horas provoca necrosis. Mientras mayor es la presión ejercida, menor es el tiempo en que se produce isquemia y posterior necrosis. Al retirar la necrosis, también llamada escara, queda la úlcera.

Corren riesgo de desarrollarlas aquellos pacientes en silla de ruedas y los que yacen en camas, tales como pacientes tetra y parapléjicos, pacientes en asilos de ancianos, y aquellos con larga estadía en los hospitales, especialmente en unidades de tratamiento o cuidado intensivo.

La localización más frecuente en pacientes parapléjicos es en el isquión y en pacientes tetrapléjicos es en la región sacra. El tratamiento quirúrgico de esta última es el motivo de esta presentación.

En nuestra casuística la úlcera por presión sacra es la de más frecuencia y cuando se presenta con daño de tejidos que abarcan desde la fascia, pasando por el músculo y llegando hasta el hueso requieren un tratamiento quirúrgico con resección del tejido alterado y reparación con colgajo musculocutáneo que, con la técnica que describimos podemos resolver, adecuadamente, esta compleja patología.

En el presente estudio se describe la técnica del colgajo miocutáneo de gluteus maximus por deslizamiento en forma triangular en $\mathrm{V}-\mathrm{Y}$, uni o bilateral para el tratamiento de úlceras por presión sacras ${ }^{1-3}$.

\section{Materiales y Método}

En este estudio retrospectivo observacional se presenta los casos de pacientes portadores de úlceras por presión sacras grado 4 en 76 pacientes y grado 5 en seis oportunidades, operados con técnica de colgajos musculocutáneo de gluteus maximus por deslizamiento en V-Y, en nuestro centro, entre el año 2011 y el 2020.

Se trata de 82 pacientes portadores de úlceras por presión a nivel sacro en que se realizó la técnica enunciada. Se analizaron las últimas 37 cirugías. Corresponden a 12 mujeres y 25 hombres, 17 parapléjicos, 12 tetrapléjicos y 8 pacientes que deambu- laban. Seis de los pacientes fueron COVID-19. Las edades fluctuaron entre 25 y 72 años. Los colgajos fueron bilaterales en 24 pacientes y unilaterales en 13. Todos usuarios de sonda Foley. Las comorbilidades fueron diabetes e hipertensión que fueron estabilizadas preoperatoriamente, por lo que no incidieron en la cirugía. El diámetro de las úlceras fluctuó entre 5 y $12 \mathrm{~cm}$. La anestesia aplicada fue mayormente general y en menor cuantía raquídea. Los exámenes realizados preoperatoriamente fueron los de rutina corrigiendo las hemoglobinas sobre $12 \mathrm{~g} \%$, la albúmina sobre $3 \mathrm{~g}$ y urocultivos negativos. Se realizó radiografía del sacro para evidenciar osteomielitis. No fue posible realizar resonancia nuclear magnética, que es el estándar de oro, por razones económicas propias de un hospital.

\section{Técnica quirúrgica (Figuras de 1 a 12)}

Bajo anestesia general o espinal los pacientes son instalados en posición prono. En la inducción anestésica se inyecta, en forma profiláctica cefazolina 1 gramo endovenoso por una sóla vez y clindamicina $900 \mathrm{mgr}$ en tres pacientes que presentaban alergia a la penicilina. Se procede a aseptizar con clorhexidina al $2 \%$. El defecto se tiñe con azul de metileno para distinguir toda la extensión de la úlcera y su bursa facilitando así su correcta y completa resección. Se infiltra los tejidos que rodean la úlcera sacra con solución con epinefrina diluida 1/1.000.000. Se reseca la cicatriz de la úlcera y la bursa efectuando hemostasia cuidadosa. Se hace la osteotomía de los procesos espinosos prominentes del sacro hasta obtener tejido óseo sangrante y de consistencia normal, asegurando así la resección de la probable osteomielitis que se corroborará con biopsia lenta. Nueva hemostasia prolija. Lavado profuso a presión con solución fisiológica al $0,9 \%$ en cantidad de tres litros a fin de arrastrar todo el material de contaminación. Se toma una muestra de tejidos blandos para cultivo cuantitativo en el que se determinará número de colonias de gérmenes por gramo de tejido.

A continuación, se diseñan, con azul de metileno, dos colgajos triangulares en "V" a ambos lados de la úlcera sacra que está en el medio, de tal forma que las bases de los triángulos quedan enfrentando la úlcera. Los bordes de los catetos de la V se infiltran con la solución antes descrita. Se inciden estos lados incluyendo la fascia y en todo el perímetro de tal modo de que quede una isla de piel triangular basando su irrigación desde profundidad a superficie a partir de vasos perforantes del músculo gluteus maximus. Cabe destacar que la porción medial de este músculo está adherido al sacro y que tanto la arteria glútea superior como la inferior tienen un pe- 


\section{ARTÍCULO ORIGINAL}

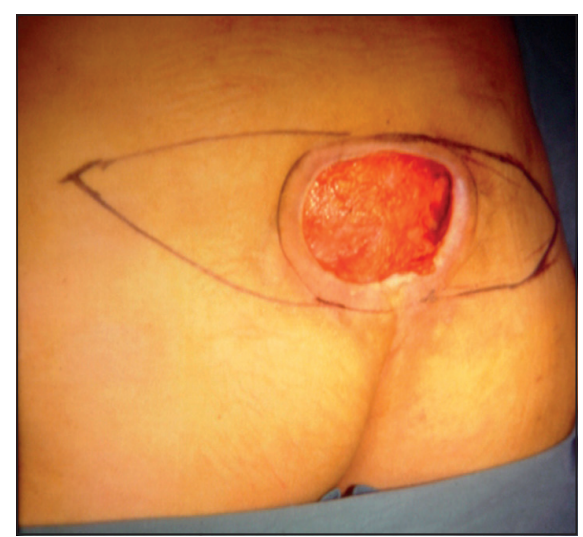

Figura 1. Úlcera por presión sacra con diseño de colgajo miocutáneo de gluteus maximus con deslizamiento en $\mathrm{V}$-Y.

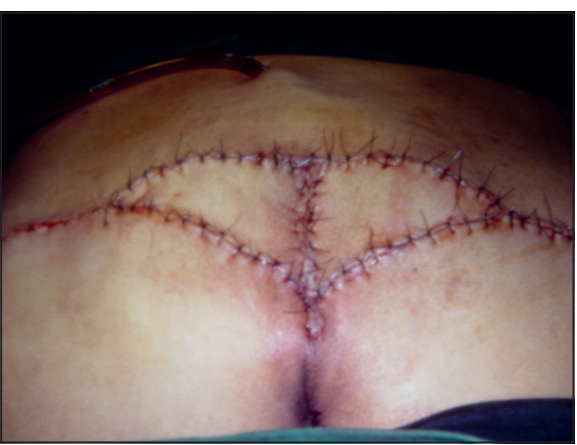

Figura 4. Posoperatorio inmediato con drenaje aspirativo y al vacío.

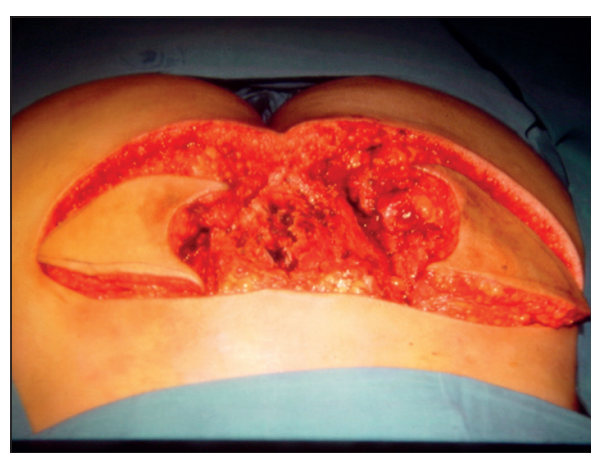

Figura 2. Incisión de la fascia en perímetro triangular del colgajo en V-Y bilateral. Las perforantes de esa isla provienen del músculo gluteus maximus. Se ha resecado las prominencias óseas sacras.

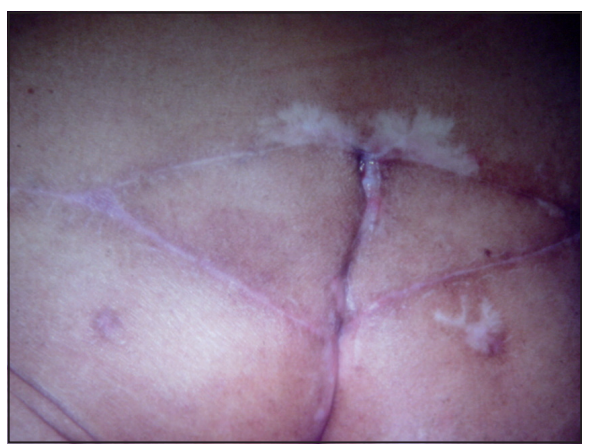

Figura 5. Posoperatorio tardío con cicatrización completa.

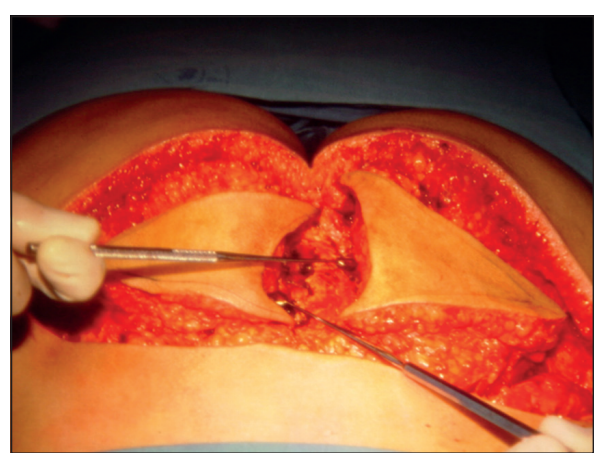

Figura 3. Avance de colgajos en isla desde lateral a medial aproximándose al colgajo opuesto. Con esta maniobra se produce un deslizamiento del músculo gluteus maximus.

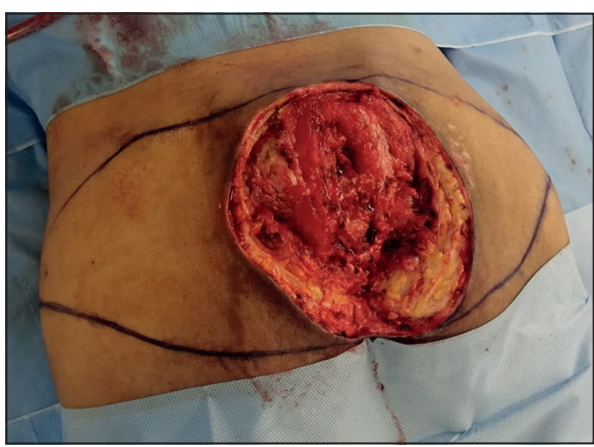

Figura 6. Aseo quirúrgico con resección de prominencias óseas sacras osteomielíticas. Diseño de colgajo en $\mathrm{V}$-Y miocutáneo de gluteus maximus en $\mathrm{V}$ - $\mathrm{Y}$.

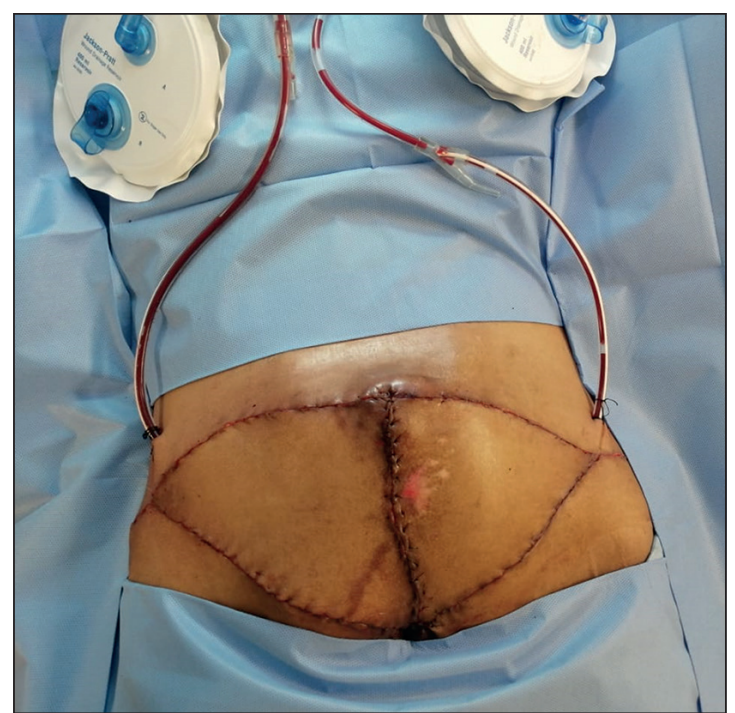

Figura 7. Posoperatorio inmediato con drenajes.

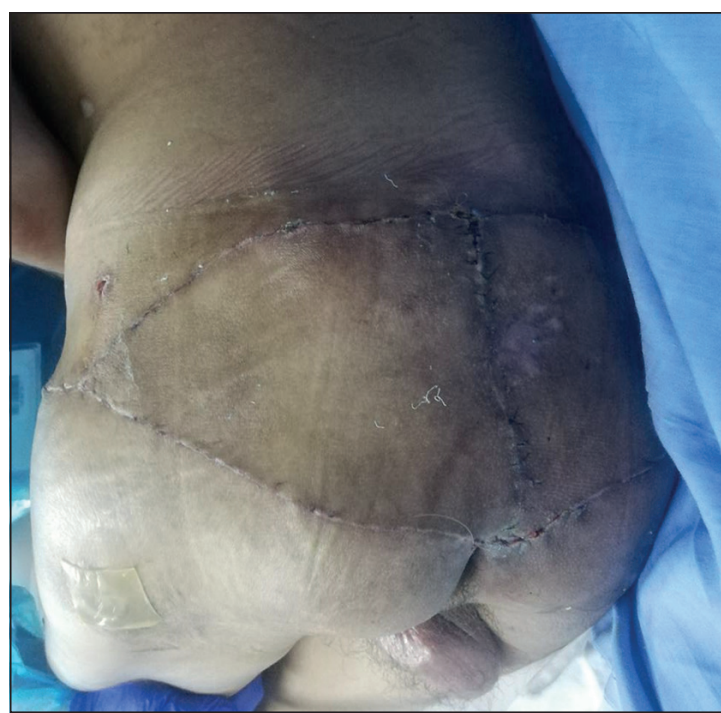

Figura 8. Posoperatorio tardío. 


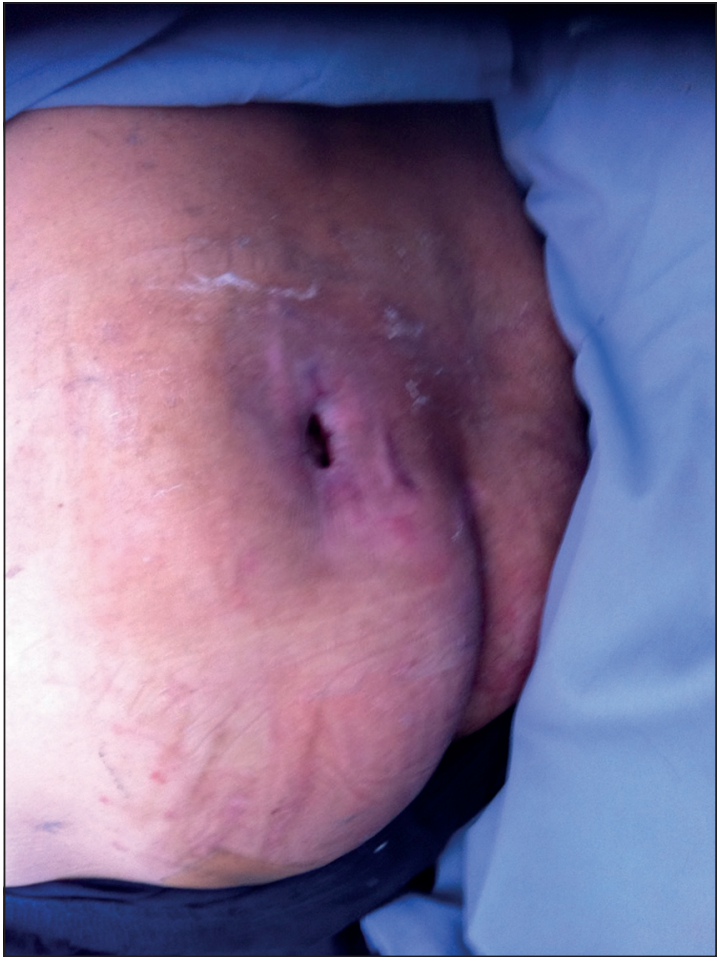

Figura 9. Úlcera por presión sacra izquierda. Existe mínima expresión cutánea externa con destrucción interna de celular subcutáneo, fascia, músculo y osteomielitis de sacro. Tipo 5.

dículo corto de $3 \mathrm{~cm}$ de largo, lo que impide mayor desplazamiento de lateral a medial, siendo la grasa, la fascia y la piel, bien perfundidas por el músculo, las que se deslizan. El complejo de la isla de piel triangular, con la grasa y fascia perfundidas por el músculo se deslizan de lateral a medial. En nuestra experiencia este avance puede ser de hasta $6 \mathrm{~cm}$ por lado, que en el caso de las úlceras de $12 \mathrm{~cm}$ la logra cubrir completamente. Con este procedimiento tampoco tuvimos necesidad se desinsertar lateralmente el gluteus maximus de la línea áspera del fémur, por lo que esta técnica también puede ser utilizada en pacientes que deambulan al no desestabilizar la cadera. Mismo procedimiento en ambos lados. Posteriormente se aproximan ambos bordes basales de este triángulo a la línea media y se suturan ambos colgajos por planos con prolene del 2/0. Piel con prolene (poliproplileno) del 3/0. Los catetos del triángulo se suturan con vicryl (poliglactina) del 3/0 y piel con monocryl (poliglecaprona) del 3/0 intradérmico. Steri Strip a la piel, curación con apósitos. Si el diámetro de la úlcera lo permite (hasta $6 \mathrm{~cm}$ ) el colgajo en V-Y puede ser sólo unilateral.

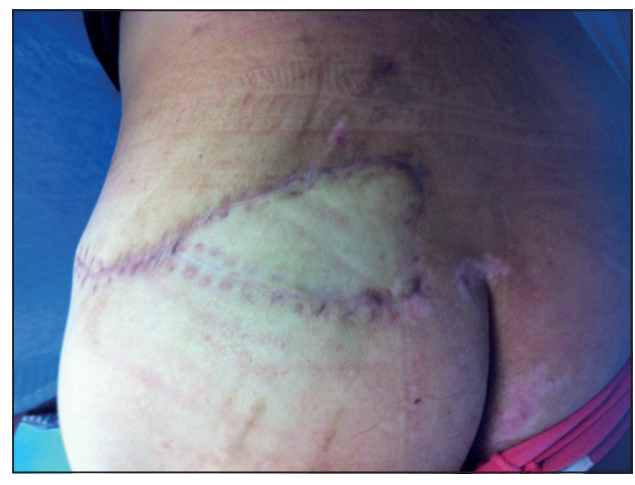

Figura 10. Posoperatorio tardío después de aseo quirúrgico, resección de piel alterada, resección de prominencias sacras osteomielíticas y avance de colgajo $V$ - $Y$ unilateral.

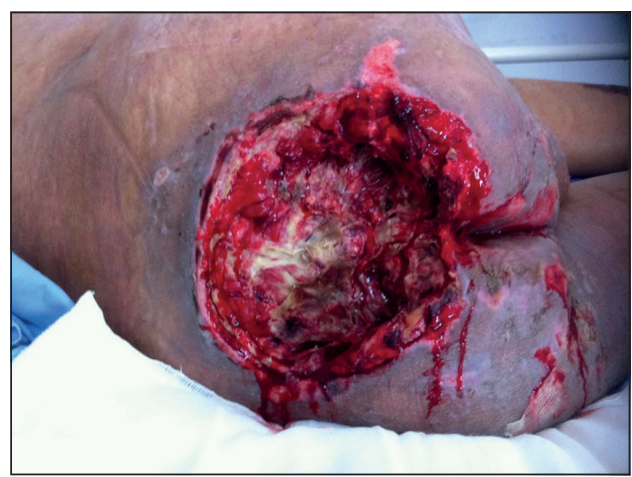

Figura 11. Aseo quirúrgico de úlcera por presión sacra con resección de prominencias óseas.

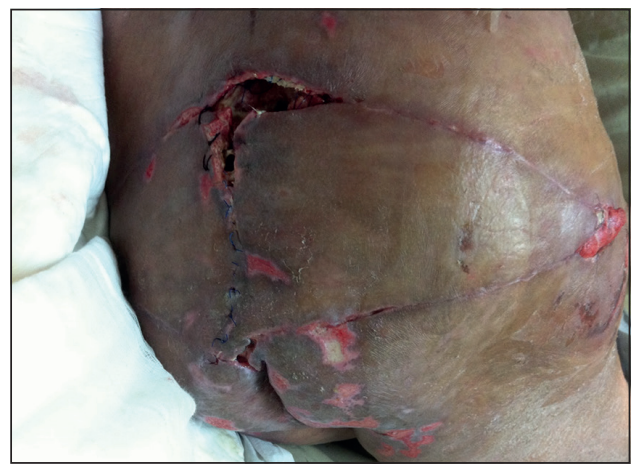

Figura 12. Colgajo miocutáneo de gluteus maximus en $\mathrm{V}$ - $\mathrm{Y}$ unilateral derecho con dehiscencia y necrosis periférica por apoyo prolongado del colgajo. Se resecó tejido sufriente y se resuturó

\section{Resultados}

La cicatrización de los colgajos fue adecuada, sin complicaciones, en 30 pacientes. Hubo dehiscencia de bordes de colgajos en 2, que fueron resuturadas sin recidiva, celulitis en 2 , tratadas con antibióticos según antibiograma, hematoma en 2 , que obligó a reoperar sin recidiva, y seroma 1 paciente, que cedió con punción. Complicaciones fueron de $16 \%$. Todos los pacientes fueron dados de alta con los colgajos cicatrizados después de 21 días, sin apoyar la zona 
operatoria. Hubo recidiva posoperatoria de la úlcera, por inadecuado cuidado en domicilio, en 5 pacientes lo que obligó a rehospitalizar y efectuar aseos quirúrgicos con posterior reavance de los colgajos con buena evolución. En las 37 cirugías analizadas la biopsia ósea diferida reveló osteomielitis en 35 de ellas.

El seguimiento fue entre 3 y 6 meses, en algunos casos dificultoso por el traslado, especialmente de los pacientes tetrapléjicos.

\section{Discusión}

Las úlceras por presión representan un grave problema de salud pública y su presencia corresponde a un indicador de calidad en las atenciones hospitalarias. Son descritas como una vulneración a los derechos humanos siendo de principal importancia su prevención destacando que es 2,5 veces más barata que su tratamiento ${ }^{4}$.

En pacientes hospitalizados su incidencia es de $10 \%$ y su prevalencia es de entre $3,5 \%$ y $29,5 \%$. Se presenta en un $23 \%$ de los pacientes parapléjicos y en un $66 \%$ de los pacientes con fracturas de fémur. La edad más frecuente es entre 71 y 90 años, 46,7\% son varones y 52,3\% mujeres. La localización mayoritaria en los hospitalizados es en la región sacra.

Con respecto a la prevención es importante destacar que los pacientes hospitalizados y que están inmovilizados por cualquiera de las causas antedichas deben ser cambiados de posición al menos cada $2 \mathrm{~h}$, evitar las fricciones y cizallamientos, lavar la piel de las zonas expuestas limpiándolas de deposiciones y orina, después secarlas y lubricarlas. El uso de colchones antiescaras debe permitir apoyos alternantes por lo que deben tener fluidos en su interior. El uso de picarones está prohibido pues si bien alivia la zona central los bordes pueden producir lesiones a la piel. En pacientes parapléjicos que usan constantemente sillas de ruedas, se debe aliviar la presión al menos cada 30 minutos, ideal cada 10 minutos, realizando el "push up", que significa apoyarse en las barandas de la silla y levantarse aliviando la presión por 2 a 3 minutos.

Para evaluar el riesgo de presentar una UPP existe la escala de Braden-Norton y Gosnell (Tabla $1)^{5}$, validada en Chile, en la cual se toma en cuenta varios parámetros que si son extremos pueden tener un alto riesgo de producción de úlceras por presión. Si la precepción sensorial local es limitada, hay exposición a la humedad constante, si el paciente está inmóvil, la nutrición es pobre y si existe peligro de roce y lesiones cutáneas el riesgo es mayor. En estos casos límites cada uno de los parámetros es asignado con un 1 , por lo tanto, el total equivale a 6 . Bajo 12 el riesgo de presentar UPP es alto.

En la producción de las úlceras por presión los factores predisponentes son la diabetes, inmunodepresión, alcoholismo, drogadicción, hipertensión, tuberculosis, cáncer, caquexia, senilidad, psicopatías, desnutrición, enfermedades vasculares, broncopulmonares y tabaquismo. Los pacientes operados en el año 2020 pertenecían todos a Fonasa A, lo que lleva a ubicarlos en un estrato socioeconómico más discreto lo que podría incidir en la producción de las úlceras sacras por los cuidados más discretos que se les pudieron otorgar (Tabla 2).

Son factores contribuyentes la movilidad reducida, inflamación local, sensibilidad disminuida o perdida, espasticidad, fricción, cizallamiento, desnutrición e higiene deficiente. En nuestra casuística desde

Tabla 1. Escala de Braden-Norton y Gosnell ${ }^{5}$. Riesgo si es menor a 12

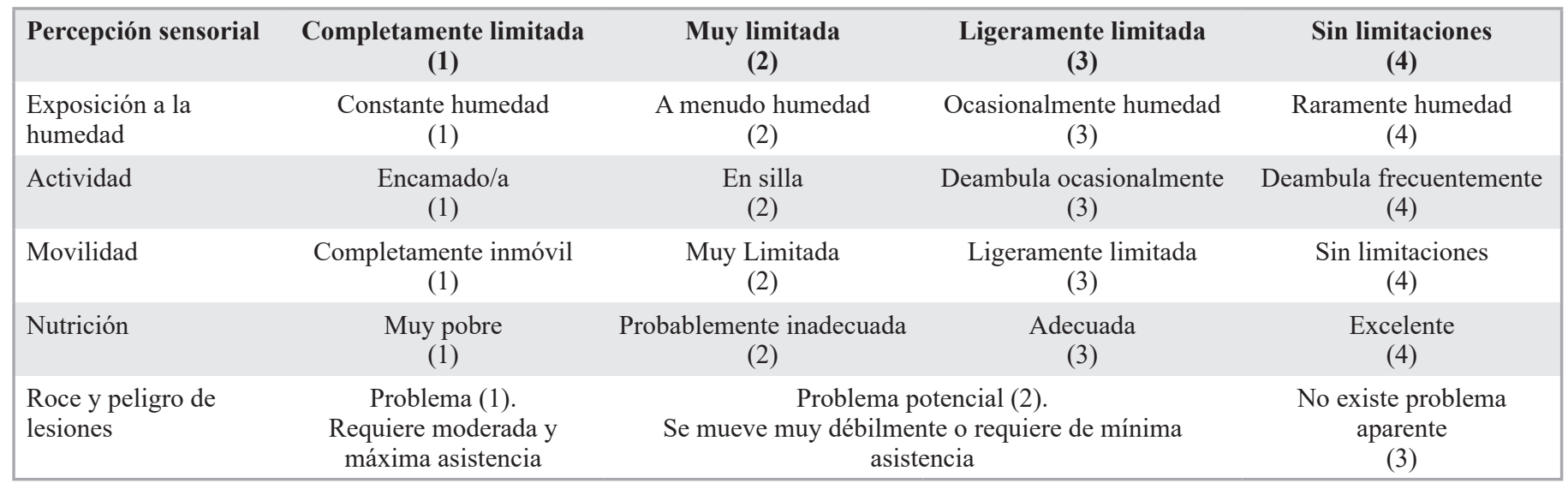


Tabla 2. Factores predisponentes a úlceras por presión sacras. Año 2020

\begin{tabular}{|lll|}
\hline Mes & Edad & Fact. predisp. \\
Marzo & 43 & Roh-Droga \\
Mayo & 26 & Droga \\
Junio & 71 & Senilidad \\
Julio & 43 & Roh-Droga \\
Julio & 64 Covid & Desnut-Roh \\
Agosto & 61 Covid & Tabaco-Desn \\
Agosto & 48 Covid & Tabaco-Drog \\
Agosto & 57 & Tabaco-Desn \\
Agosto & 58 & Senilidad \\
Septiembre & 56 Covid & Roh-Tabaco \\
\hline
\end{tabular}

marzo a septiembre de 2020, época de COVID-19, hubo diez pacientes operados con esta técnica en los cuales los factores predisponentes más importantes fueron el alcoholismo, drogadicción, senilidad, desnutrición y tabaquismo que incidieron en la producción de este tipo de úlcera al alterar la circulación de la piel y los planos más profundos (Tabla 3). En los 4 pacientes COVID-19 el factor contribuyente más frecuente fue la movilidad reducida con compresión de la zona sacra y los factores predisponentes más frecuentes fueron el alcoholismo, el tabaco, la droga y la desnutrición (Tabla 4).

La ubicación de las úlceras por presión son múltiples y pueden presentarse en todas las ubicaciones en que existen prominencias óseas como, por ejemplo, la región sacra.

La localización más frecuente en pacientes parapléjicos es el isquión y en pacientes tetrapléjicos es en la región sacra, motivo esta última, de esta presentación.

Las úlceras por presión sacras se pueden producir a diferentes niveles de sufrimiento de la piel. Grado 1: eritema y exposición de la dermis. Grado 2: exposición del celular subcutáneo. Grado 3: hasta la fascia y músculo. Grado 4: compromiso del hueso y finalmente una, 5, que tiene mínima exteriorización de la piel pero que tiene destrucción subcutánea de celular subcutáneo, fascia, músculo y compromiso del hueso. En este último caso se debe resecar la piel comprometida y reparar la lesión subyacente.

En los grados 1 y 2 el tratamiento es conservador con curación avanzada de heridas, es decir curaciones húmedas con geles, hidrogeles y apósitos específicos. En los grados 3, 4 y 5 el tratamiento es quirúrgico.
Tabla 3. Características socioeconómicas y enfermedades concomitantes. Año 2020

\begin{tabular}{|llll|}
\hline Mes & Edad & Socecon. & Comorb. \\
Marzo & 43 & Fonasa A & No \\
Mayo & 26 & Fonasa A & No \\
\hline Junio & 71 & Fonasa A & Hipert \\
Julio & 43 & Fonasa A & No \\
Julio & 64 COVID & Fonasa A & Hipert \\
Agosto & 61 COVID & Fonasa A & Diabet \\
\hline Agosto & 48 COVID & Fonasa A & No \\
Agosto & 57 & Fonasa A & Hipert \\
Agosto & 58 & Fonasa A & Diabet \\
Septiembre & 56 COVID & Fonasa A & Diabet \\
\hline
\end{tabular}

Tabla 4. Pacientes operados de UPP sacras desde marzo a septiembre de 2020

\begin{tabular}{|lcccc}
\hline Mes 2020 & Edad & Colgajo V-Y & COVID-19 & Plejia \\
Marzo & 43 & Doble & No & Tetraplejia \\
Mayo & 26 & Doble & No & Tetraplejia \\
Junio & 71 & Doble & No & No \\
Julio & 43 & Doble & No & No \\
Julio & 64 & Derecho & Sí & No \\
Agosto & 61 & Derecho & Sí & No \\
Agosto & 48 & Doble & Sí & No \\
Agosto & 57 & Derecho & No & No \\
Agosto & 58 & Derecho & No & No \\
Septiembre & 56 & Doble & Sí & No \\
\hline
\end{tabular}

Previo a este tratamiento quirúrgico es indispensable que todos los parámetros estén normales, es por ello que la hemoglobina debe ser superior a $12 \mathrm{~g}$, el consumo de proteínas debe estar de 1 a $1,5 \mathrm{~g}$ por kilo por día, con una albúmina sobre $3 \mathrm{~g}$. Dado que la mayoría de nuestros pacientes presentan daño medular irreversible es que se deben eliminar los espasmos flexores, previamente, con baclofeno, toxina botulínica, alcoholización o fenolización o rizotomía de los nervios de los músculos flexores de las extremidades inferiores. Descartar infecciones urinarias, pielonefritis, microvejiga y litiasis renal que son frecuentes por el uso de sonda Foley o auto cateterización y que pueden producir bacteremia o septicemia que infecta los colgajos. Para determinar la presencia de osteomielitis se puede realizar una 
radiografía del sacro, pero el mejor método para detectarla es una resonancia nuclear magnética ${ }^{6-9}$.

Cabe destacar que el uso de colgajo musculocutáneo es el más adecuado dada su excelente irrigación ${ }^{9}$ que permite rellenar el defecto y aportar mayor cantidad de oxígeno, nutrientes, y antibióticos ${ }^{10}$. El hecho que se realice con forma de V-Y ${ }^{11}$ permite el fácil deslizamiento de lateral a medial. El éxito de este tipo de colgajos nos ha llevado a describirlos, también, como tratamiento en úlceras por presión a nivel trocantérico e isquiático ${ }^{12-14}$. En lesiones masivas de la región sacra y perineal se describe el uso de colgajos musculares o miocutáneos con microcirugía ${ }^{15}$, hecho que en nuestra casuística no ha sido necesario.

Como toda técnica quirúrgica esta no está exenta de complicaciones que están descritas en otros trabajos ${ }^{16}$ y que también se presentaron en nuestros pacientes y que correspondieron a dehiscencias, infecciones, hematomas, seromas, y sufrimiento de colgajos marginales; en ningún caso observamos pérdidas completas.

El tratamiento quirúrgico expuesto corresponde a un exhaustivo desbridamiento a nivel de la úlcera sacra resecando la prominencia ósea de los procesos con posterior reparación. El colgajo utilizado es un músculo cutáneo de gluteus maximus, número 3 de la clasificación de Mathes y Nahai ${ }^{7}$, con una isla de piel triangular cuya base está orientada a nivel de la úlcera. Esta isla de piel es escindida y desplazada de lateral a medial sin necesidad de desinsertar el músculo de la línea áspera del fémur, lo que cobra relevancia en el sentido de que este colgajo puede ser utilizado también en pacientes que deambulan al no desestabilizar la cadera. Si es bilateral los colgajos son suturados entre sí, y si es unilateral es suturado al borde de la úlcera ya desbridada. La irrigación de la isla de piel triangular proviene de perforantes provenientes del músculo gluteus maximus desplazada de lateral a medial por deslizamiento.

Esta técnica de colgajos en isla triangular con avance es conocida como de V-Y, pues se avanza como $\mathrm{V}$ y es suturada como $\mathrm{Y}$. Es una técnica de relativa fácil ejecución, confiable, de resultados previsiblemente adecuados y seguros. Puede ser realizada por cirujanos con entrenamiento no sólo en cirugía plástica sino también en cirugía general lo que la hace ser susceptible de ser aplicada en cualquier región del país.

Una alternativa válida para cubrir estas úlceras sacras grado 4 y 5 es el colgajo de rotación, siempre musculocutáneo, de gluteus maximus pero pensamos que el colgajo V-Y tiene la ventaja de mayor desplazamiento que puede cubrir úlceras de mayor diámetro y sin el riesgo de desprender la piel del músculo lo que haría que el colgajo sufriese. Otras alternativas son los colgajos romboidales tipo Random, como el Dufourmentel y el Limberg y también los fasciocutáneos y la ventaja de los miocutáneos estriba en que dan mayor volumen para cubrir úlceras profundas, proveen mayor flujo sanguíneo aportando más oxígeno, más nutrientes y antibióticos.

\section{Conclusión}

El tratamiento quirúrgico de la cobertura de úlceras por presión sacras grado 3, 4 y 5 con colgajo miocutáneo de Gluteus maximus con isla de piel en $\mathrm{V}-\mathrm{Y}$, por deslizamiento, ha sido satisfactorio para nuestros pacientes con un porcentaje de complicaciones bajos.

Es un procedimiento bien reglado, seguro, reproducible, aplicable a cualquier úlcera sacra con grados profundos en pacientes con daño medular, parapléjicos y tetrapléjicos, y pacientes que caminan. Es por esto último que ha sido muy significativo que hemos podido operar pacientes con COVID-19 que son pacientes que deambulan y con técnica similar en ambos casos.

\section{Responsabilidades éticas}

Protección de personas y animales. Los autores declaran que para esta investigación no se han realizado experimentos en seres humanos ni en animales.

Confidencialidad de los datos. Los autores declaran que en este artículo no aparecen datos de pacientes.

Conflictos de interés: no hay. 


\section{Bibliografía}

1. Ramirez OM, Hurwitz DJ, Futrell JW. The expansive Gluteus Maximus Flap. Plast Reconstr Surg. 1984;74:769-70.

2. Ramirez OM, Orlando JC, Hurwitz DJ. The Sliding Gluteus Maximus Myocutaneous Flap: Its relevance in ambulatory patients. Plast Reconstr Surg. 1984;74:68-75.

3. Pu LL. Reconstruction of a large gluteal soft-tissue defect with the double-opposing V-Y fasciocutaneous advancement flap. Plast Reconstr Surg. 2007;119:599-603.

4. Chavarría G, Cruz L. Úlceras por presión: Manejo quirúrgico en pacientes de un Centro Nacional de Rehabilitación. Cir Plast Iberolanamer. 2002;28:63-8.

5. Blümel M, Tirado K, Schiele C, Schönffeldt G, Sarrá S. Validez de la escala de Braden para predecir úlceras por presión en población femenina. Rev Med Chile. 2004;132:595-600.

6. Lobos L. Úlceras por presión. En Juri A, editor. Cirugía Plástica. Santiago de Chile: Tangram Ediciones; 2001:673-89.
7. Mathes SJ, Nahai F. Posterior Trunk: Gluteus Maximus-Gluteal Thigh Flap. En Mathes SJ, Nahai F, editors. Reconstructive Surgery: Principles, Anatomy and Technique. New York: Churchill Livingstone; 1997:499-13.

8. Hester TR, Schneider WJ, Hill HL. Pressure Sores: Reconstruction.

En Mathes SJ, Nahai F, editores. Clinical applications for muscle and musculocutaneous flaps. Segunda Edición. St Louis, Missouri: The C.V. Mosby Company; 1982:454-89.

9. Ramirez OM. Blood supply to the gluteus maximus flap. Plast Reconstr Surg. 2000;105:812-13.

10. Calderon W, Chang N, Mathes SJ. Comparison of the effect of bacterial inoculation in musculocutaneous and fasciocutaneous flaps. Plast Reconstr Surg. 1986;77:785-94.

11. Andrades PR, Calderon W, Leniz P, Bartel G, Danilla S, Benitez S. Geometric analysis of the V-Y advancement flap and its clinical applications. Plast Reconstr Surg. 2005; 115:1582-90.

12. Calderón W, Oyarse E, Olivares C, Roco H, Leniz P, Calderón D, et al.
Cobertura de úlceras por presión trocantéricas con colgajo de Tensor de fascia lata en V-Y. Rev Chil Cir. 2009;61:429-32.

13. Llanos S, Calderón W, Piñeros JL, Roco H, Roa R, Leniz P, et al. Colgajo miocutáneo de glúteo mayor en isla con cierre $\mathrm{V}$ en $\mathrm{Y}$ para la cobertura de úlceras isquiáticas. Cir Plást Iberolatinamer. 2006;32.:41-8

14. Calderón W, Andrades P, Leniz P, Piñeros JL, Llanos S, Roa R, Irribarren $O$. The cone flap: a new and versatile fasciocutaneous flap. Plast Reconstr Surg. 2004;114:1539-42.

15. Vogt PM, Kall S, Lahoda LU, Spies M, Muehlberger T. The free "mutton chop" flap: a fascio-musculocutaneous flap for the reconstruction of the entire sacral and perineal area. Plast Reconstr Surg. 2004;114:1220-4.

16. Sameem M, Au M, Wood T, Farrokhyar F, Mahoney J. A systematic review of complication and recurrence rates of musculocutaneous, fasciocutaneous, and perforator-based flaps for treatment of pressure sores. Plast Reconstr Surg. 2012;130:67e-77e. 